\title{
A low-kickback-noise and low-voltage latched comparator for high-speed folding and interpolating ADC
}

\author{
Guohe Zhang ${ }^{\text {a) }}$, Bo Wang, Feng Liang, and Zhibiao Shao \\ Dept. of Microelectronics, Xi'an Jiaotong University, Xi'an, Shaanxi, 710049, China \\ a)guohe.z@gmail.com
}

\begin{abstract}
A novel low-kickback-noise Class-AB latched comparator utilizing unilateralization technique is presented for high-speed folding and interpolating analog-to-digital converter (ADC). The load transistors are independent with the clock signal. So the comparator can suppress the kickback noise significantly and work at low power supply. Dummy transistors and neutralization technique are also introduced to further reduce the noise. The comparator is simulated in $0.18-\mu m$ standard digital CMOS technology. A very low kickback noise voltage of $0.14 \mathrm{mV}$ is realized at the differential input voltage of $300 \mathrm{mV}$. The power dissipation is about $202 \mu \mathrm{W}$ at $1.8 \mathrm{~V}$ supply voltage, $250 \mathrm{MHz}$ clock frequency.
\end{abstract}

Keywords: latched comparator, kickback noise, analog-to-digital converters

Classification: Integrated circuits

\section{References}

[1] H. Lee, "A 12-b $600 \mathrm{ks} / \mathrm{s}$ digitally self-calibrated pipelined algorithmic ADC," IEEE J. Solid-State Circuits, vol. 29, no. 4, pp. 509-515, April 1994.

[2] Y. Wang and B. Razavi, "An 8-bit 150-MHz CMOS A/D converter," IEEE J. Solid-State Circuits, vol. 35, no. 3, pp. 308-317, March 2000.

[3] S. Kim and M. Song, "An 8-bit 200MSPS CMOS A/D converter for analog interface module of TFT-LCD Driver," Proc. IEEE Int. Symp. Circuits Syst., Sydney, NSW, pp. 528-531, May 2001.

[4] P. Amaral, J. Goes, and N. Paulino, "An improved low-voltage low-power CMOS comparator to be used in high-speed pipeline ADCs," Proc. IEEE Int. Symp. Circuits Syst., Phoenix, AZ, USA, pp. 141-144, May 2002.

[5] K. Uyttenhove and M. S. J. Steyaert, "A 1.8 V 6-bit 1.3-GHz flash ADC in 0.25 CMOS," IEEE J. Solid-State Circuits, vol. 38, no. 7, pp. 1115-1122, July 2003.

[6] P. M. Figueiredo and J. C. Vital, "Kickback Noise Reduction Techniques for CMOS Latched Comparators," IEEE Trans. Circuits Syst., vol. 53, no. 7, pp. 541-545, July 2006. 


\section{Introduction}

Class-AB latched comparator can be introduced into the folding and interpolating analog-to-digital converter (ADC) for high speed and low power dissipation. However, the kickback noise can not be neglected. Due to the rail-to-rail signals at the regeneration nodes coupled directly to the inputs, it may make the ADC output code false and degrade the resolution efficiency. Fig. 1 (a) shows a traditional Class-AB latched comparator structure. Several approaches have been reported to reduce the kickback noise of the Class-AB latched comparator. Source followers are effective to reduce the kickback noise but induce more static consumption [1]. Neutralization technique can accomplish low kickback noise in some extent [2]. During the regeneration phase, the input nodes can be isolated from the regeneration nodes by using switches [3]. However, the input voltage is still disturbed when the sampling switches close. The isolation between the drains of differential pair transistors and the regeneration nodes reduces the kickback noise $[4,5]$. However, it will make the input transistors go into the triode region easily, and the drain voltage variations will originate kickback noise in evidence. An improved circuit proposed by Figueiredo [6] can suppress the kickback noise significantly, as shown in Fig. 1(b). Nevertheless, the circuit still needs to be improved further because there is still large kickback noise when transistors $M_{11} / M_{12}$ are transiting between cutoff and saturation.

In this paper, a low kickback noise Class-AB latched comparator is proposed using unilateralization and neutralization techniques. Preamplifier and dummy MOS transistors are also introduced into the circuit to suppress the kickback noise. The comparator is simulated in 0.18- $\mu \mathrm{m}$ standard digital CMOS technology. A very low kickback noise voltage of $0.14 \mathrm{mV}$ is realized at the differential input voltage of $300 \mathrm{mV}$. Under the $250 \mathrm{MHz}$ clock frequency, low power dissipation can be achieved at $1.8 \mathrm{~V}$ supply voltage.

\section{Proposed Class-AB latched comparator}

Fig. 1(a), (b) and (c) shows the traditional Class-AB latched comparator, an improved Class-AB latched comparator proposed by Figueiredo, and the propose Class-AB latched comparator in this paper respectively. Compared to the former two structures, we focused on suppressing the kickback noise in the circuit.

In the input stage of Fig. 1 (c), $M_{1}$ and $M_{13}, M_{2}$ and $M_{14}$ form two pairs of source-coupled amplifiers, which can restrain the voltage variations at the sources of $M_{1} / M_{2}$. The diode-connected load transistors $M_{11} / M_{12}$ operate in saturation region, not controlled by the clock signal in order to avoid the appearance of large instantaneous current. When the signal clk is low (reset phase), the differential input signals are amplified and applied to $V_{o p}$ and $V_{o n}$. When clk goes high, nodes $a_{1} / a_{2}$ are disconnected from regeneration nodes. The latch circuit starts regenerating rail-to-rail output signals, which could not affect nodes $a_{1} / a_{2} . M_{15} / M_{16}$ are used to reduce the clock feedthrough effects of $M_{9} / M_{10} . M_{17}$ and $M_{18}$ can neutralize the voltage variations at the 




(a)

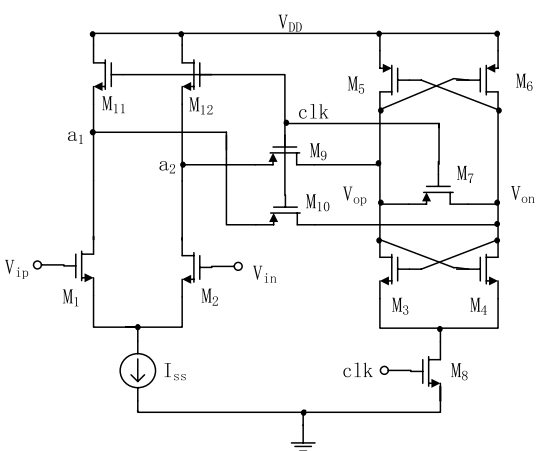

(b)

$V_{D D}$

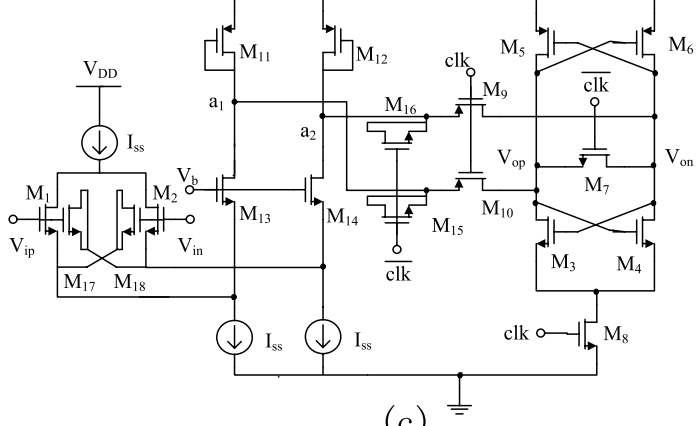

(c) $\stackrel{1}{=}$

Fig. 1. (a) Traditional Class-AB latched comparator, (b) An improved Class-AB latched comparator proposed by Figueiredo [6], (c) The proposed Class$\mathrm{AB}$ latched comparator in the paper

sources of $M_{1} / M_{2}$. The changement in our circuit compared to Fig. 1 (b) can suppress the kickback noise significantly, which will be illuminated later.

Considering the low supply voltage application, a source-coupled amplifier is used to achieve unilateralization. This can limit the direction of the signalflowing which just flows from the inputs to the nodes $a_{1}$ and $a_{2}$. Therefore, the voltage variations at nodes $a_{1} / a_{2}$ is coupled to the inputs more slightly. Although the circuit would draw more current, lower supply voltage makes the whole comparator consume less power. The NMOS input transistors are designed to reduce parasitic capacitance of the input transistors to relieve the couple effects.

It is assumed that the voltage at node $a_{1}$ changes $\Delta V$, the voltage variation $\Delta V_{s}$ at the source of $M_{1}$ is given by

$$
\Delta V_{s}=\Delta V \frac{r_{o 1}}{\left[1+\left(g_{m 13}+g_{m b 13}\right) r_{o 13}\right] r_{o 1}+r_{o 13}} \approx \frac{\Delta V}{\left(g_{m 13}+g_{m b 13}\right) r_{o 13}}
$$

where, $g_{m 13}$ and $g_{m b 13}$ are respectively the transconductance and body transconductance of $M_{13} \cdot r_{o 1} / r_{o 13}$ are the output resistances of $M_{1} / M_{13}$. Usually, $\left(g_{m 13}+g_{m b 13}\right) r_{o 13}>10$, so the coupling effects between $a_{1,2}$ and sources of $M_{1,2}$ can be reduced significantly, resulting in much lower kickback noise.

Diode-connected transistors are proposed as the loads of the input different pair. Firstly, they needn't switch between cutoff and saturation, avoiding large instantaneous current and large voltage variation at $a_{1} / a_{2}$. Thus, low kickback noise can be achieved. Secondly, the current flows through the load 
transistors $M_{11} / M_{12}$ in Fig. 1 (c) during the whole clock period. The transistors $M_{11} / M_{12}$ in Fig. 1 (b) are cutoff during half of the clock period. It looks like that the power dissipation would increase. Actually, when $M_{11} / M_{12}$ cut off, $M_{5}$ and $M_{6}$ serve as the loads of the preamplifier, and hence there is still current in preamplifier. The power dissipation also remains. So the power dissipation in the proposed circuit doesn't increase in fact.

To ensure the small voltage variations at $a_{1} / a_{2}$, the load resistance of preamplifier should change as slightly as possible during the whole clock period. When clk is high, $M_{11}$ and $M_{12}$ serve as load and their resistances are defined as

$$
R_{\text {high }} \approx \frac{1}{g_{m 11,12}}
$$

When clk is low, $M_{5}$ and $M_{11}, M_{6}$ and $M_{12}$ are parallel connected as loads. The total load resistance is given as

$$
R_{\text {low }} \approx \frac{1}{g_{m 5,6}+g_{m 11,12}}
$$

So we design large $g_{m 11,12}$ and small $g_{m 5,6}$ to diminish the difference between $R_{\text {high }}$ and $R_{\text {low }}$ to reduce voltage variation at $a_{1} / a_{2}$. However, too large $g_{m 11,12}$ may cause low gain of preamplifier, reducing the resolution and speed of the comparator. And, too small $g_{m 5,6}$ would slow the latch. To make a compromise here, the size of $M_{11} / M_{12}$ was about fourfold as that of $M_{5} / M_{6}$.

In addition, when clk is low, switch $M_{7}$ closes and $V_{o p}$ and $V_{o n}$ are reset. There will be ill-fitting voltage variation at $a_{1} / a_{2}$ with inconsistent $V_{o p}$ and $V_{\text {on }}$. Thus, proper size of $M_{3} / M_{4}$ and $M_{5} / M_{6}$ will be designed to ensure that the value of Vop and Von are close to the static operation point at $a_{1} / a_{2}$.

The dummy transistors $M_{15}$ and $M_{16}$ are added to offset the clock feedthrough effects, which makes voltage at $a_{1} / a_{2}$ vary suddenly when clk switches. The signal clk is coupled to the nodes $a_{1} / a_{2}$ through the gatesource capacitances of $M_{7} / M_{8}$ while the $\overline{c l k}$ is coupled to $a_{1} / a_{2}$ through gate capacitances of $M_{15}$ and $M_{16}$. According to

$$
V_{c l k} \frac{0.5 W_{9,10} C_{o v}}{0.5 W_{9,10} C_{o v}+W_{15,16} C_{o v}}-V_{c l k} \frac{W_{15,16} C_{o v}}{0.5 W_{9,10} C_{o v}+W_{15,16} C_{o v}}=0
$$

where $\mathrm{W}$ and $\mathrm{L}$ is the width and length of MOS transistors, $C_{o v}$ is gate overlap capacitance per width, and $V_{c l k}$ is the voltage amplitude of clk. The clock feedthrough effects can be minimized by setting $W_{15,16}=0.5 W_{9,10}, L_{15,16}=$ $L_{9,10}$. Furthermore, the PMOS switch $M_{7}$ in Fig. 1 (b) was substituted to NMOS switch. So the NMOS switch can be controlled by $\overline{c l k}$ and minimize the clock feedthrough effects.

When the source voltage of $M_{1} / M_{2}$ varies, preamplifier provides the charge current for the parasitic capacitance of $M_{1} / M_{2}$. Those charge currents will cause the kickback noise. As shown in Fig. 1 (c), two capacitances equal to the value of gate-source capacitance of $M_{1} / M_{2}$ are added to reduce the kickback noise. Because the currents are produced when the gate capacitance of $M_{17} / M_{18}$ discharges, two capacitances equal to the gate-source capacitance of $M_{1} / M_{2}$ can be added to reduce the kickback noise as shown in Fig. 1 (c). 


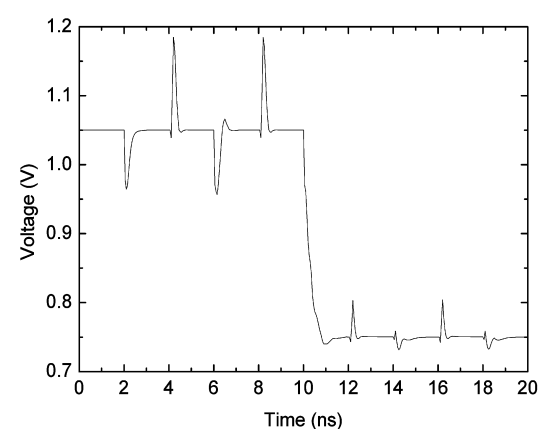

(a)

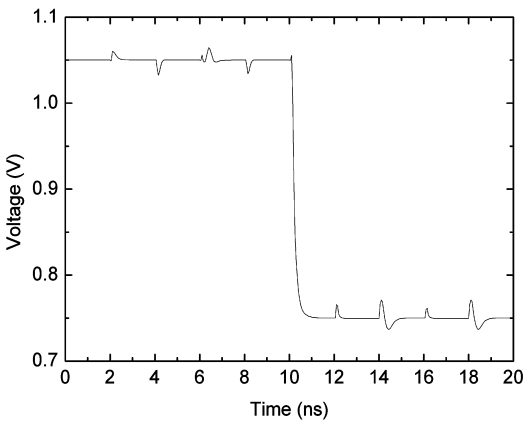

(c)

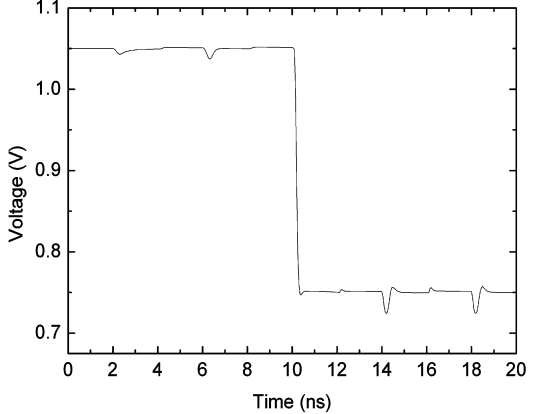

(b)

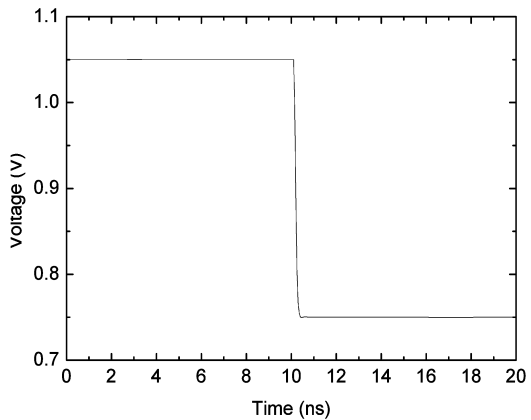

(d)

Fig. 2. The kickback noise from (a) the Fig. 1(b) circuit, (b) improved circuit only use unilateralization technology, (c) improved circuit only use the load transistors independent with clock signal, (d) The proposed circuit

Table I. Summary of comparator's performances

\begin{tabular}{ccc}
\hline & This work & {$[6]$} \\
\hline Kickback noise $(\mathrm{mV})$ & 0.14 & 4 \\
Power supply $(\mathrm{V})$ & 1.8 & 1.8 \\
Power dissipation $(\mu W)$ & 202 & 268 \\
Clock frequency $(\mathrm{MHz})$ & 250 & 200 \\
Technology $(\mu m)$ & 0.18 & 0.18 \\
\hline
\end{tabular}

\section{Simulation results}

The proposed comparator are simulated in $0.18-\mu m$ standard digital CMOS technology at $1.8 \mathrm{~V}$ supply voltage using HSPICE. The clock frequency is $250 \mathrm{MHz}$. The kickback noise is obtained by measuring the gate voltage of input transistors. An $8 \mathrm{k} \Omega$ resistance is inserted between the gates of input transistors and the signal source during the measurements.

The kickback noises in Figueiredo's circuit and ours are compared when the amplitude of input differential voltage is $300 \mathrm{mV}$. Fig. 2 (a) indicates that the comparator in the Fig. 1 (b) almost has no kickback noise during the regeneration phase. However, when clk is switching, it is still large kickback noise, about $136 \mathrm{mV}$. The kickback noise is about $25 \mathrm{mV}$ only using unilateralization technique, as shown in Fig. 2 (b). The load transistors proposed here can also considerably reduce the kickback noise to $27 \mathrm{mV}$, as shown in 
Fig. $2(\mathrm{c})$. Fig. $2(\mathrm{~d})$ shows the kickback noise in our proposed comparator. The value is just about $0.14 \mathrm{mV}$. It can be seen that the kickback noise was suppressed significantly here. Furthermore, the power dissipation of the whole comparator including the output latch is just about $202 \mu \mathrm{W}$. So, the circuit can work at lower supply voltage to improve the power efficiency further.

Table I summarizes a comparison on the performances with the comparator proposed by Figueiredo. According to simulation results of Hspice, our comparator can work at higher frequency but consume less power. Importantly, the kickback noise voltage is very low, just $4 \%$ of that of comparator in $[6]$.

\section{Conclusion}

An improved low kickback noise and low voltage Class-AB latched comparator is discussed. The inputs and the regeneration nodes are isolated using the unilateralization technique. The source-coupled amplifier can work at low power supply. The load transistors independent with clock signal are introduced to avoid large instantaneous current. So the kickback noise is reduced significantly. In addition, the dummy transistors relieve the clock feedthrough effects and neutralization technique also helps to reduce further the kickback noise. According to simulation results, the comparator achieves a very low kickback noise voltage of $0.14 \mathrm{mV}$ at the differential input voltage of $300 \mathrm{mV}$. Low power dissipation of $202 \mu \mathrm{W}$ can be achieved at $1.8 \mathrm{~V}$ supply voltage while the clock frequency is increased to $250 \mathrm{MHz}$. 